frequency radio waves, and sferics are not observed by satellites. But there are special circumstances where energy can propagate through in the so-called 'whistler mode'. These conditions, as measured by other instruments on the Orbiter, are thought to occur fairly frequently on the night side of Venus. When they do, the plasma-wave detector sees impulsive signals of a kind not normally encountered at orbital altitude. The instrument would need much better time resolution to obtain the length or shape of the pulses, but they are consistent with an origin from lightning and no other explanation has been suggested. Taken all together, the evidence for lightning on Venus is fairly convincing.
Ksanfomaliti also suggests that lightning could generate a mean nightside brightness $10^{-4}$ that of the day side. This source is put forward as an explanation of the 'ashen light', a phenomenon reported by many visual observers who see the whole disk faintly illuminated when the planet is in a crescent phase. It remains to be seen whether such suggestions and reports can be reconciled with the brightnesses observed from vehicles close to Venus.

The indications are that lightning is likely to occur in any substantial planetary atmosphere. Theories of electrification are faced with the need to explain its presence under a wide variety of circumstances and atmospheric compositions.

\title{
Paradoxical prolactin
}

\section{from Alan S. McNeilly}

LACTATION in many mammals is associated with suppression of ovulation as a result of the failure of ovarian follicles to develop to the appropriate stage. The widely varying suckling patterns in different societies (from very frequent short periods, for example in the !Kung peoples of the Kalahari, to infrequent longer periods of suckling) are all associated with suppression of normal gonadotropin secretion and of ovarian activity, and with raised basal levels of prolactin which increase further in response to the suckling stimulus (see McNeilly Br. Med. Bull. 35, 151; 1979). As ovarian activity normally resumes once suckling ceases, either the suckling stimulus itself and/or the raised levels of prolactin may be responsible for the suppression of gonadotropin secretion and the consequent failure of normal follicular development.

The best evidence that the prolactin level itself may be responsible for ovulatory inhibition comes from cases of pathological hyperprolactinaemia which is the apparent cause of $20 \%$ of secondary amenorrhoeas. Unlike lactation, the raised prolactin levels in this condition may be due to an alteration in hypothalamic catecholamine turnover. The way in which prolactin itself may play a part has received scant attention; it may act on the hypothalamic-pituitary axis to suppress gonadotropin secretion, or may act directly on the ovary to suppress follicular development.

Evidence that prolactin is implicated in normal follicle development has been obtained from studies of progesterone

Alan McNeilly is a Research Scientist at the $M R C$ Unit of Reproductive Biology, Edinburgh. secretion from human granulosa cells in vitro (McNatty, Sawers \& McNeilly Nature $250,653 ; 1974)$. Levels of prolactin equivalent to those seen during the normal menstrual cycle were essential to maintain LH-stimulated progesterone secretion. In contrast, high levels of prolactin equivalent to those present in lactation and pathological hyperprolactinaemia inhibited progesterone secretion, an inhibition which could not be overcome by the addition of gonadotropins (LH or FSH). A similar situation was apparent in the mouse where follicular progesterone secretion in vitro was also inhibited by high levels of prolactin (McNatty, Neal \& Baker $J$. Reprod. Fertil. 37, 155; 1976).

But how prolactin can have both an obligatory permissive role in progesterone secretion and an inhibitory effect remains unexplained. Studies by Veldhuis and Hammond (this issue of Nature, page 262) go some way to resolving this dilemma. Using short term in vitro studies of progesterone secretion from porcine granulosa cells they found that oestradiol $17 \beta$ seems to modify the action of prolactin.

Previous studies suggest that development from a primordial to a Graafian (preovulatory) follicle is initially under the control of FSH and oestradiol. LH stimulates oestradiol and androgen secretion from the thecal layer of cells surrounding the developing follicle; FSH stimulates aromatisation of these androgens to oestrogens by the granulosa cells within the developing follicle. Both FSH and oestradiol are required for the proliferation of granulosa cells and antrum formation within the developing follicle and induce an increase in $\mathrm{LH}$ receptors on these granulosa cells. Prolactin is detectable within follicular fluid of all follicles and receptors have been detected on granulosa cells. Veldhuis and
Hammond found that progesterone secretion from granulosa cells in vitro taken from small immature pig follicles could be inhibited by prolactin. Short-term oestradiol treatment also suppressed progesterone secretion. However prolonged treatment with oestrogens (for $48 \mathrm{~h}$ ) resulted in increased progesterone secretion and reversed the action of prolactin from inhibition to synergistic stimulation of progesterone. The authors conclude therefore that "oestrogens may regulate the divergent actions of prolactin in the mammalina ovary."

The basis of the oestrogen requirement for proliferation and maturation of granulosa cells is as yet unknown. Thus the change in response to prolactin seen in the present experiments may simply reflect the maturation of granulosa cells: prolactin will stimulate progesterone secretion from granulosa cells taken from large mature pig follicles. In this respect the effects of prolactin in the pig follicle may be different from those in the human where high levels of prolactin inhibit progesterone secretion from granulosa cells regardless of the stage of follicular development.

The importance of the direct inhibitory effects of high prolactin levels on steroidogenesis by granulosa cells in follicles before ovulation is emphasised by the fact that the rare cases of ovulation in hyperprolactinaemia, in particular that associated with lactation, is associated with an inadequate corpus luteum capable of secreting only limited amounts of progesterone (McNeilly, Howie \& Houston, unpublished data). This suggests that prolactin-mediated inhibition of normal granulosa cell development before ovulation may explain the inadequacy of luteal function. This is supported by the observation that drug-induced hyperprolactinaemia in the luteal phase has only a slight effect on progesterone secretion from the developed corpus luteum whereas hyperprolactinaemia induced during the follicular phase results in the formation of an inadequate corpus luteum (Delvoye et al. C. r. Seanc. hebd. Acad. Sci. (Paris) Ser D. 279, 1463; 1974). McNatty (Fertil. Steril. 32,$433 ; 1979$ ) has provided more direct evidence in support of this. Raised levels of prolactin in plasma and ovarian follicular fluid were associated with a reduced number of granulosa cells and a marked reduction in intrafollicular steroidogenesis, not always apparent from the levels of circulating oestrogens.

Thus it seems that prolactin may have an important regulatory role in granulosa cell development within the follicle. Although present reports suggest that oestrogens may modulate the action of prolactin, the species difference in this action has yet to be resolved. The importance of understanding these mechanisms is apparent since hyperprolactinaemic blockade of steroidogenesis may in part explain the natural contraceptive effect of lactation in women and many other mammals. 